\title{
Characterization and Optimization of Four-Wave-Mixing Wavelength Conversion System
}

Kaminski, Pawel Marcin; Da Ros, Francesco; Porto da Silva, Edson; Pu, Minhao; Yankov, Metodi Plamenov; Semenova, Elizaveta; Yvind, Kresten; Clausen, Anders Thomas; Forchhammer, Søren; Oxenløwe, Leif Katsuo

Total number of authors:

11

Published in:

Journal of Lightwave Technology

Link to article, DOI:

10.1109/JLT.2019.2933226

Publication date:

2019

Document Version

Peer reviewed version

Link back to DTU Orbit

Citation (APA):

Kaminski, P. M., Da Ros, F., Porto da Silva, E., Pu, M., Yankov, M. P., Semenova, E., Yvind, K., Clausen, A. T., Forchhammer, S., Oxenløwe, L. K., \& Galili, M. (2019). Characterization and Optimization of Four-Wave-Mixing Wavelength Conversion System. Journal of Lightwave Technology, 37(21), 5628-5636.

https://doi.org/10.1109/JLT.2019.2933226

\section{General rights}

Copyright and moral rights for the publications made accessible in the public portal are retained by the authors and/or other copyright owners and it is a condition of accessing publications that users recognise and abide by the legal requirements associated with these rights.

- Users may download and print one copy of any publication from the public portal for the purpose of private study or research.

- You may not further distribute the material or use it for any profit-making activity or commercial gain

- You may freely distribute the URL identifying the publication in the public portal 


\title{
Characterization and Optimization of Four-Wave-Mixing Wavelength Conversion System
}

\author{
P.M. Kaminski, F. Da Ros, E.P. da Silva, M. Pu, M.P. Yankov, E. Semenova, K. Yvind, A.T. Clausen, \\ S. Forchhammer, L.K. Oxenløwe, and M. Galili
}

\begin{abstract}
In this work, we present a comprehensive experimental and numerical investigation of the impact of system parameters on wavelength converters based on four-wavemixing, with focus on practical system implementations in addition to the interaction within the nonlinear medium. The input signal power optimization is emphasized according to the trade-off between the linear and the nonlinear impairments, and the origin of the limitations at the optimum is studied. The impact of the input signal quality on the converted idler is discussed, and depending on the dominant noise contribution a varying conversion penalty is demonstrated. The penalty is also shown to scale with increasing number of WDM channels due to additional nonlinear cross-talk between them. Finally, by means of numerical simulations we extend the experimental characterization to high pump powers, showing the impact of parametric noise amplification, and different pump laser linewidths, which lead to increased phase-noise transfer. The experimental characterization employs an integrated AlGaAson-insulator waveguide, and the numerical simulations accompany the results to make the analysis general for $\chi^{(3)}$ materials that satisfy the assumptions of the split-step Fourier method.
\end{abstract}

Index Terms - four-wave mixing, integrated waveguides, quadrature amplitude modulation, coherent communications.

\section{Introduction}

$\mathrm{P}$ RESENT-day telecommunication systems are continuously facing new challenges in terms of throughput and cost-efficiency, as a result of the ever-increasing traffic demands [1]. All-optical signal processing (OSP) techniques are being applied to address these problems, while also providing new functionalities to future networks. In particular, optical wavelength conversion has the potential to facilitate traffic grooming and resolve wavelength contention, improving on overall spectrum utilization [2]. It can be easily implemented by means of four-wave mixing in $\chi^{(3)}$ media, offering low latency and power consumption, broadband operation and transparency to modulation formats and data rates [3]-[7]. Integrated nonlinear platforms for FWM have recently been attracting increasing

P.M. Kaminski, F. Da Ros, M. Pu, E. Semenova, K. Yvind, A.T. Clausen, S. Forchhammer, L.K. Oxenløwe and M. Galili are with the Department of Photonics Engineering, Technical University of Denmark, Kongens Lyngby, 2800 Denmark, e-mail: pkam@fotonik.dtu.dk.

M.P. Yankov is with the Department of Photonics Engineering, Technical University of Denmark, Kongens Lyngby, 2800 Denmark, and with Fingerprint Cards A/S, Herlev, 2730 Denmark, e-mail: meya@fotonik.dtu.dk.

E.P. da Silva was with the Department of Photonics Engineering, Technical University of Denmark, Kongens Lyngby, 2800 Denmark. $\mathrm{He}$ is now with the Department of Electrical Engineering of the Federal University of Campina Grande (UFCG), Paraiba, Brazil, email: edson.silva@dee.ufcg.edu.br. attention due to high nonlinearity and small footprint for compact solutions [3]-[10]. Among them, AlGaAs-oninsulator $(\mathrm{AlGaAsOI})$ is characterized by low propagation losses and tunable bandgap to provide two-photon absorption (TPA) free operation around $1550 \mathrm{~nm} \mathrm{[6]-[10].} \mathrm{In}$ addition to evident benefits of wavelength conversion from the network perspective, the generated wavelength-shifted idler can also be exploited to compensate the inherent fiber nonlinearity by means of optical-phase conjugation (OPC), leading to increased total system throughput [11] or extended transmission reach [12]. In either case, the signal-to-idler conversion is typically performed within the network, with degraded input signals far from transmitter and receiver. However, wavelength converters have so far been primarily characterized for ideal inputs with maximum optical signal-to-noise ratio (OSNR), and noise loading at the receiver side, as in [4]-[6] and [13]-[16]. The impact of input signal quality has only been preliminary analyzed in [17] for FWM applied to format conversion, and in [15] with regard to OSNR saturation. Similar metric has been employed in [13], where the conversion noise figures were calculated from input-to-output OSNR differences, while in [18] the excess noise of a simplified setup was estimated using electrical spectrum analyzer, but with limited system optimization. The other relevant work has only focused on the FWM process alone, and in [19], [20] theoretical noise figures of wavelength converters have been discussed for quantum limited signals. Further characterizations on the noise sources and the achievable noise figures have been carried out for fiber optical parametric amplifiers (FOPA) in [19]-[24], yet they can only be partially translated to wavelength converters which are based on the same principle, but operate in a different system configuration. To our knowledge, no systematic characterization of wavelength conversion systems with regard to the optimum performance and the principal design requirements has been reported yet.

In this paper, we extend our previous characterization of [25], where increasing conversion penalty was demonstrated for high OSNR inputs, to a thorough experimental and numerical analysis of a $\chi^{(3)}$ wavelength converter. Instead of only characterizing the nonlinear medium, we focus on the complete system and study the origins of impairments associated with the entire conversion process. We illustrate the impact of the input signal OSNR on the generated idler, provide insight into signal power and pump optimization for maximum performance, and 
relate the conversion penalty to the number of wavelengthdivision-multiplexed (WDM) channels. Most importantly, unlike in [13], OSNR is no longer used as the quality metric of the system, and instead we turn to effective received signal-to-noise ratio (SNR) estimated directly from the transmitted and received symbol sequence in order to account for the different types of impairments due to conversion. Finally, we outline the trade-offs between the linear and the nonlinear penalties of a practical system, and discuss the balance between them for best conversion performance. The experimental investigation employs 16-quadrature amplitude modulation (QAM) signals at $16-\mathrm{GBd}$ in an AlGaAsOI-based wavelength converter, while the numerical analysis relies on the splitstep Fourier method (SSFM) to validate and generalize the trends [26], [27].

The paper is organized as follows. In Section II, the general conversion setup is introduced and the characterization methodology is given. Then in Section III, the specific system implementation is provided for both the experimental setup and the numerical simulations. The impact of input signal quality on the generated idler is evaluated in Section IV, and the consequence of increasing number of the converted WDM channels is discussed in Section V. Numerical simulations extend the experimental characterization in terms of higher pump power and different pump linewidths in Sections VI and VII, respectively. Finally, the paper is concluded in Section VIII.

\section{Conversion Setup and Methodology}

Regardless of the specific application, every FWMbased wavelength converter consists of three main building blocks: FWM stage for idler generation, signal and pump suppression, and idler amplification, as sketched in Fig. 1. Such systems have been implemented experimentally and emulated numerically [4]-[6], [11]-[16]. It is noted that $\chi^{(2)}$-based wavelength converters [28]-[30] require a similar setup, obey analogous procedure, and can also be modelled with the proposed scheme. However, they differ in terms of the physical interactions and limitations, and therefore the analysis results cannot be transferred directly.

In this analysis, data signals with a specific total input OSNR of all channels $\left(\mathrm{OSNR}_{i n}\right)$ and total input signal power $\left(\mathrm{P}_{i n}\right)$, are mixed with a continuous wave pump, and injected into a nonlinear medium. The pump power $\left(\mathrm{P}_{p}\right)$ is typically high to achieve significant conversion efficiency $(\mathrm{CE})$, and it is placed around the zero-dispersion wavelength (ZDW) of the nonlinear structure for broadbandwidth operation [3]-[6]. At the output of the nonlinear medium, the total OSNR of all channels of the generated idler $\left(\mathrm{OSNR}_{\text {out }}\right)$ is measured, and it is selected with appropriate filtering. Finally, it is amplified to a fixed output power with an erbium-doped fiber amplifier (EDFA) at the output of the converter. The penalties associated with the conversion therefore do not only originate from the FWM-process itself, but also from the inherent propagation/filtering losses and amplified

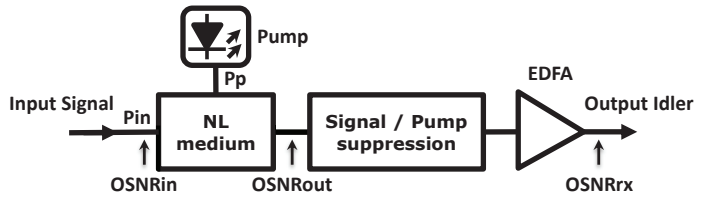

Fig. 1. Schematic diagram of a FWM-based wavelength converter.

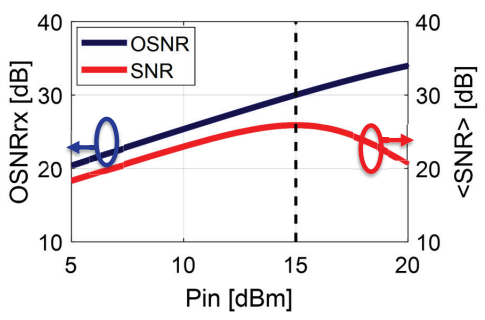

Fig. 2. Numerically simulated input power $\mathrm{P}_{i n}$ optimization with respect to received idler OSNR (blue) and SNR (red) for a single channel and $9 \mathrm{~mm}$ waveguide at $\mathrm{P}_{p}=20 \mathrm{dBm}$ and $\mathrm{OSNR}_{i n}=40 \mathrm{~dB}$. The optimum launch power is marked in black.

spontaneous emission (ASE) noise due to the EDFA. At the converter output, the total idler OSNR of all channels $\left(\mathrm{OSNR}_{r x}\right)$ is measured, and the idler is received with a coherent setup followed by digital signal processing, including adaptive equalization, carrier phase recovery and effective received SNR estimation. SNR is estimated on per channel basis directly from the transmitted and received QAM symbols, $x_{k}$ and $y_{k}$ respectively, after all DSP blocks from a sequence of $K$ symbols $k=[1 ; K]$ as $<\mathrm{SNR}>=\mathrm{E}_{k}\left[\left|x_{k}\right|^{2}\right] / \mathrm{E}_{k}\left[\left|y_{k}-x_{k}\right|^{2}\right]$, where $\mathrm{E}[\cdot]$ denotes the expectation operation, as in [31].

OSNR is no longer the quality metric as it only accounts for the ASE noise, but neglects other types of impairments, e.g. phase noise and nonlinearity. Therefore, it may not accurately reflect the performance of a complex nonlinear communication system. Instead, the wavelength converter is evaluated in terms of effective received SNR, and the general relation between the idler SNR and OSNR at the receiver is illustrated in Fig. 2 for varying $\mathrm{P}_{i n}$. Increasing the input signal power leads to increased idler power at the waveguide output, reducing the required EDFA amplification and the impact of the ASE noise of the system, as will be discussed more thoroughly in Section IV. Consequently, the OSNR increases linearly with $\mathrm{P}_{i n}$. On the other hand, high input power in the nonlinear structure causes stronger distortions due to selfphase modulation (SPM), cross-phase modulation (XPM) and intra-channel FWM, ultimately limiting the received idler quality and the effective SNR. As a result, for specific system parameters there exists an optimal input power $\mathrm{P}_{\text {in }}$ which maximizes the received idler SNR. These trends are consistent with the preliminary analysis of [5] and [6], where similar power dependence have been demonstrated for the Q-factor and the achievable information rate (AIR) for inputs with maximum OSNRin, respectively. 

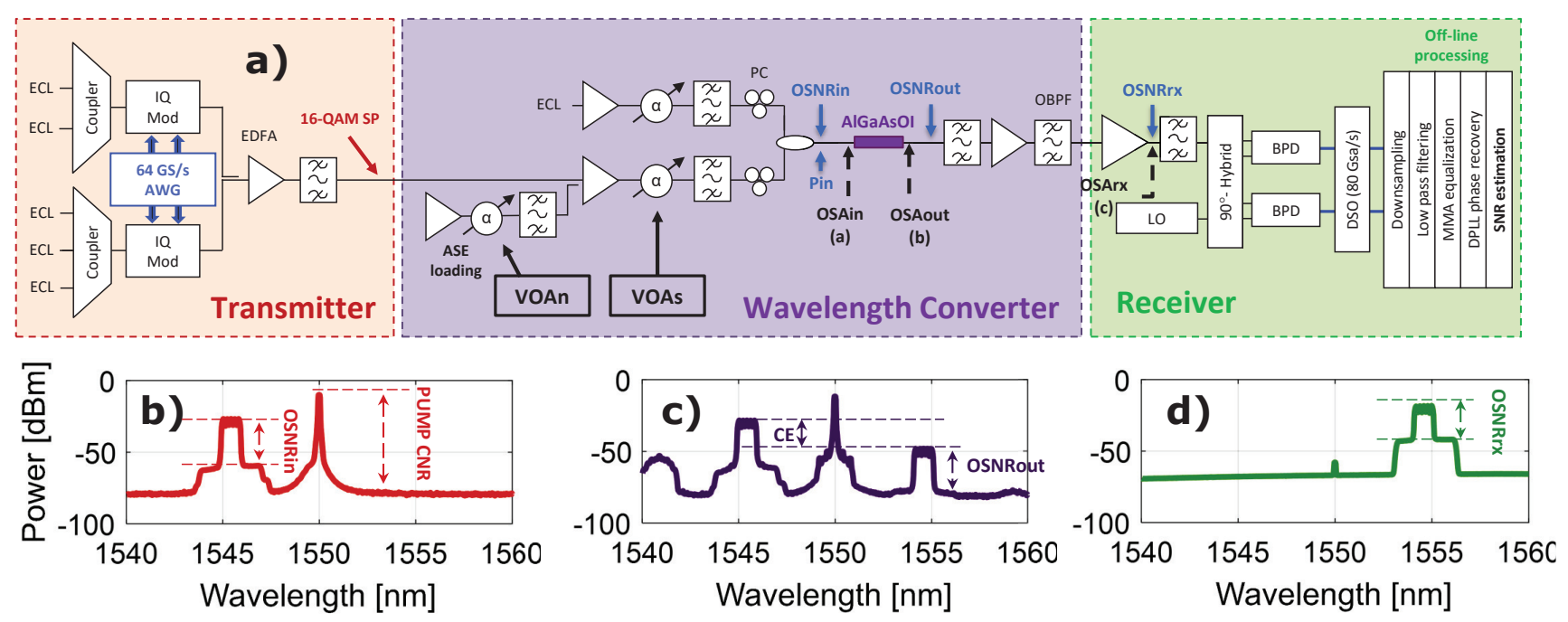

Fig. 3. Experimental setup for wavelength converter characterization (a) with the corresponding spectra at the waveguide input (b), waveguide output (c), and receiver (d) with $5 \mathrm{WDM}$ channels and $9 \mathrm{~mm}$ waveguide at $20 \mathrm{dBm}$ pump power, maximum OSNR $\mathrm{m}_{\text {in }}$ and optimum signal launch power.
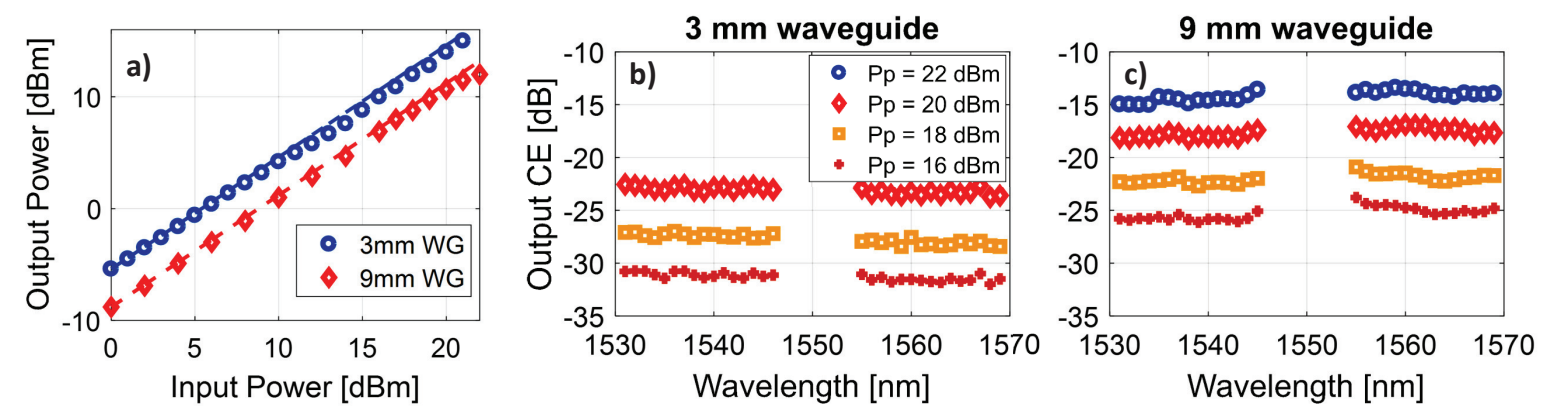

Fig. 4. Experimental characterization of 3 and $9 \mathrm{~mm}$ AlGaAsOI waveguides employed for the analysis: measured (points) and linear (dashed) loss in (a), output conversion efficiency for 3 and $9 \mathrm{~mm}$ waveguides in (b) and (c), respectively.

\section{Specific System Implementation}

The detailed experimental implementation of the setup is given in Fig. 3(a). WDM signals on a 25-GHz grid are generated by external cavity lasers (ECLs), with 16-QAM data at rate $16-\mathrm{GBd}$ modulated onto the corresponding waves with external IQ modulators (one for even and one for odd channels). The channels are then coupled together, amplified with an EDFA, and launched into a wavelength converter based on degenerated FWM in AlGaAsOI [6], [25]. The OSNR evolution is tracked and measured throughout the system by means of the recorded spectra as indicated: $\mathrm{OSNR}_{\text {in }}$ in Fig. 3(b), OSNR ${ }_{\text {out }}$ in Fig. 3(c), and $\mathrm{OSNR}_{r x}$ in Fig. 3(d). Signal power $\mathrm{P}_{\text {in }}$ into the converter is varied with a variable optical attenuator (VOA) along the signal path, and OSNR in is controlled by loading ASE noise from an extra EDFA. Before entering the nonlinear medium, the signal is combined with a continuous-wave (CW) pump at $1550 \mathrm{~nm}$ from a sub$\mathrm{kHz}$ linewidth fiber laser. The study is conducted for waveguide lengths of 3 and $9 \mathrm{~mm}$, as indicated later. Both signal and pump are co-aligned to the waveguide TE-mode with polarization controllers (PC) for minimum propagation loss and maximum FWM. At the output of the waveguide, the generated idler is selected with optical tunable filters (OTF), and it is amplified to a fixed output power and received. The system is reproduced in numerical simulations based on asymmetric SSFM with a constant step size to verify and extend the analysis for the parameters difficult to vary experimentally. The input signal is controlled with respect to $\mathrm{P}_{i n}$ and $\mathrm{OSNR}_{i n}$, and the pump characteristics are specified by pump power $\left(\mathrm{P}_{p}\right)$, carrier-to-noise ratio (CNR) (see Fig. 3(b)) and linewidth $\left(\nu_{p}\right)$. The simulations adopt the linear loss and the nonlinearity (CE) of the AlGaAsOI waveguides, neglecting the nonlinear absorption and the wavelength dependence of CE: propagation loss $\alpha=0.8 \mathrm{~dB} / \mathrm{mm}$; nonlinearity $\gamma=0.351 / \mathrm{W} / \mathrm{mm}$; dispersion $D=1.64 * 10^{-4}$ $\mathrm{ps} / \mathrm{nm} / \mathrm{mm}$; dispersion slope $S=0$. The parameters are consistent with the experimental characterization in Fig. 4. For both waveguide lengths, the total output power scales linearly with the total input power up to $22 \mathrm{dBm}$ (Fig. 4(a)), thus the nonlinear loss is negligible. The corresponding output $\mathrm{CE}$ extends uniformly beyond the C-band, and it increases quadratically with pump power up to $-23 \mathrm{~dB}$ at $\mathrm{P}_{p}=20 \mathrm{dBm}$ for $3 \mathrm{~mm}$ waveguide (Fig. 4(b)) and $-15 \mathrm{~dB}$ at $\mathrm{P}_{p}=22 \mathrm{dBm}$ for $9 \mathrm{~mm}$ waveguide (Fig. 4(c)). In the end, effective SNR is again employed 


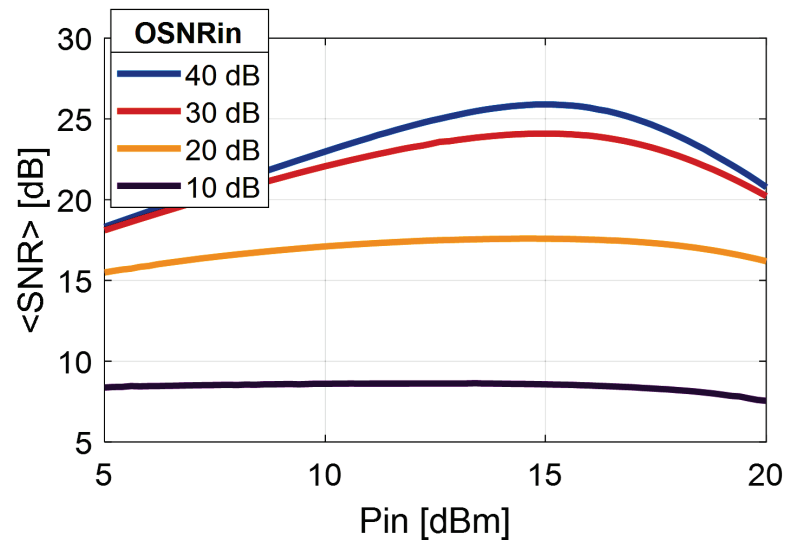

Fig. 5. Numerically estimated idler SNR and input power optimization for varying $\mathrm{OSNR}_{i n}$ for a single channel and $9 \mathrm{~mm}$ waveguide at $\mathrm{P}_{p}=20 \mathrm{dBm}$.

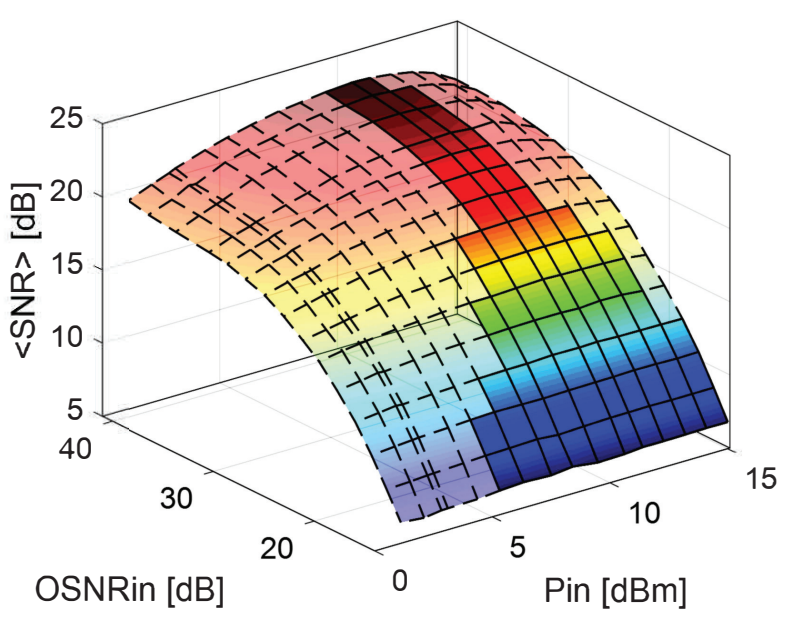

Fig. 6. Experimentally measured idler SNR for varying input signal OSNR $_{i n}$ and $\mathrm{P}_{i n}$ at $\mathrm{P}_{p}=22 \mathrm{dBm}$ with five WDM channels and $9 \mathrm{~mm}$ waveguide. The highlighted region denotes the optimum input powers that fall within $0.2 \mathrm{~dB}$ of the maximum SNR at a fixed $\mathrm{OSNR}_{i n}$.

to evaluate the performance of the system, with all the measurements conducted for the central channel only.

\section{Impact of the Input Signal Quality}

Input signal degradations influence the conversion operation and the idler quality, and are of practical importance for wavelength conversion or phase conjugation processes. In order to evaluate such an impact, the input signal OSNR $_{i n}$ is varied and the input signal power $\mathrm{P}_{\text {in }}$ is optimized for each value with respect to the estimated idler SNR in both numerical simulations and laboratory experiment. It should be emphasized that the aim is not to reproduce the exact experimental measurements with the numerical simulations, but rather compare them and validate the obtained trends. The SNR results are illustrated in Fig. 5 for the numerical analysis. As discussed beforehand, there exists an optimum $\mathrm{P}_{\text {in }}$ that maximizes the received idler SNR, and it is limited by the linear noise from one side, and the nonlinearity from the other. The

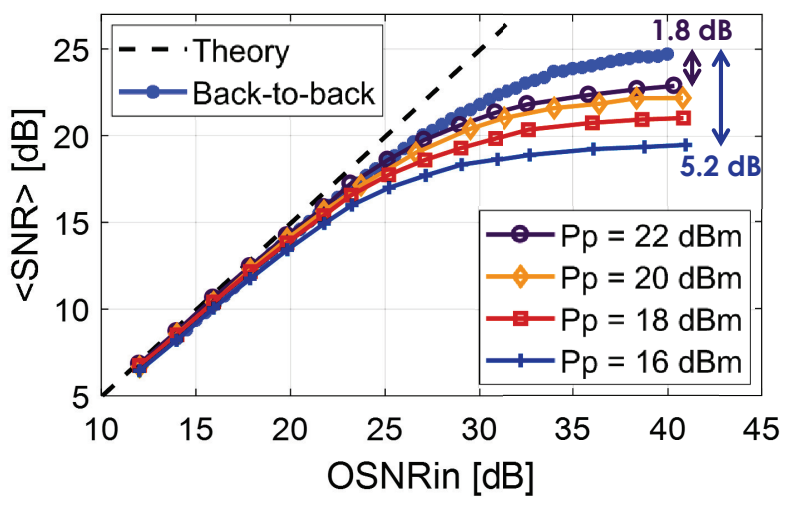

Fig. 7. Experimentally measured maximum idler SNR at optimum $\mathrm{P}_{i n}$ as a function of $\mathrm{OSNR}_{i n}$ for different $\mathrm{P}_{p}$ with five WDM channels and $9 \mathrm{~mm}$ waveguide.
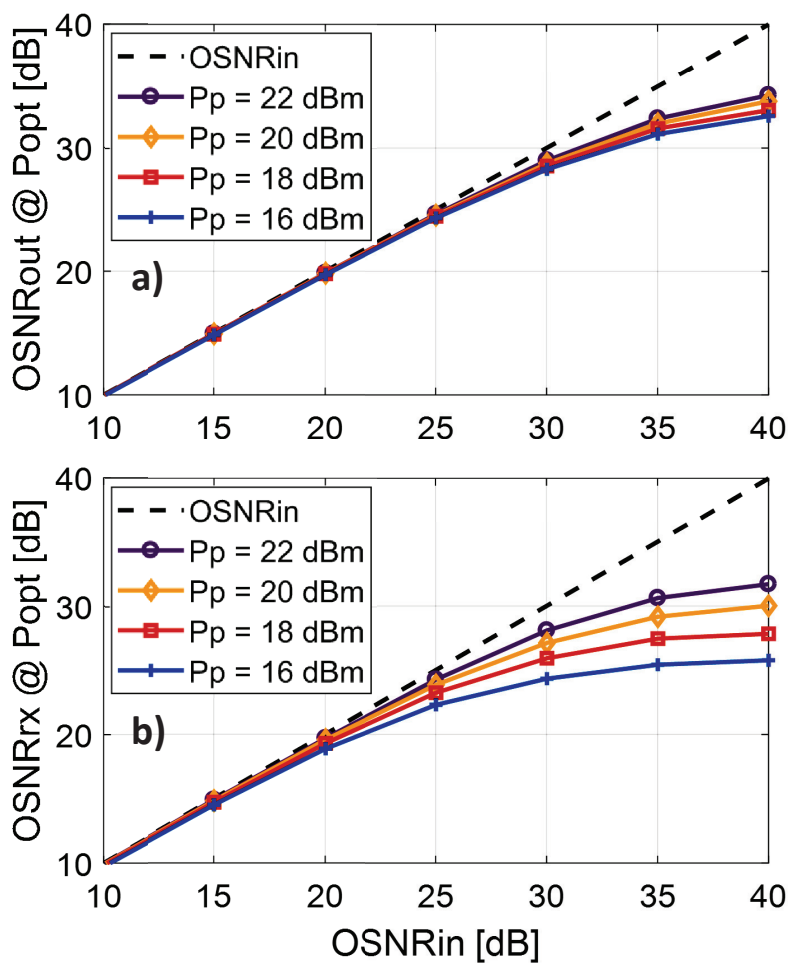

Fig. 8. Simulated idler OSNR at optimum $\mathrm{P}_{\text {in }}$ at the waveguide output (a) and the receiver (b) versus $\mathrm{OSNR}_{i n}$ for varying $\mathrm{P}_{p}$ with a single WDM channel and $9 \mathrm{~mm}$ waveguide.

effective SNR generally increases with $\mathrm{OSNR}_{i n}$, and its variation with respect to $\mathrm{P}_{\text {in }}$ is more significant for high OSNR $_{\text {in }}$ inputs, leading to a clear optimum, but becomes lower as $\mathrm{OSNR}_{\text {in }}$ decreases. For signals dominated by the ASE noise at the waveguide input, the degradations due to conversion are insignificant compared to the noise already present, thus resulting in nearly flat SNR curves.

The corresponding experimental characterization on the impact of the input signal quality is given in Fig. 6, and clearly follows the numerical trends, i.e. SNR increase with $\mathrm{OSNR}_{i n}$, and a higher variation with respect to $\mathrm{P}_{\text {in }}$ for high-quality inputs. The optimum signal launch powers at a given $\mathrm{OSNR}_{\text {in }}$ are highlighted, assuming 

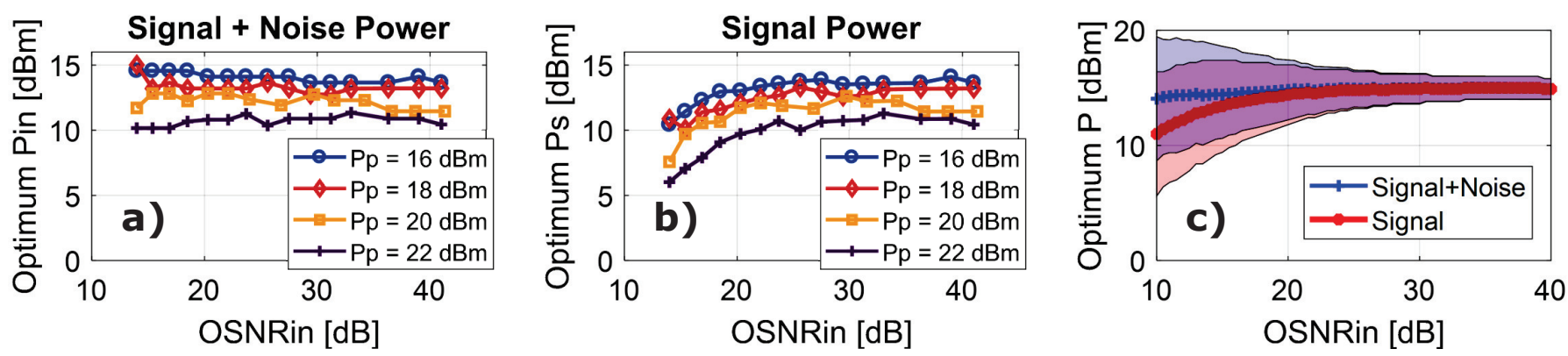

Fig. 9. Experimentally measured optimum total input signal power $\mathrm{P}_{i n}$ (a) and optimum signal power $\mathrm{P}_{s}$ (b) versus $\mathrm{OSNR}_{i n}$ for varying $\mathrm{P}_{p}$ with five WDM channels and $9 \mathrm{~mm}$ waveguide. (c) Numerically simulated optimum powers at $\mathrm{P}_{p}=20 \mathrm{dBm}$ with $9 \mathrm{~mm}$ waveguide and a single channel for reference, optimum power tolerance within $0.2 \mathrm{~dB}$ SNR offset marked alongside.

$0.2 \mathrm{~dB}$ SNR tolerance from the maximum value. It is apparent that the input power optimization is particularly important at high $\mathrm{OSNR}_{i n}$, and becomes less relevant as the input noise starts to dominate. With $\mathrm{P}_{\text {in }}$ fixed to the optimum, the obtained SNR versus $\mathrm{OSNR}_{i n}$ is plotted for a number of pump powers in Fig. 7. The conversion results are benchmarked against a back-to-back SNR reference for unconverted signal measured at the waveguide input with pump off, and the theoretical SNR maximum for signals limited by additive white Gaussian noise (AWGN) [32]. The performance of the wavelength converted idler approaches the back-to-back reference and the theoretical maximum at low $\mathrm{OSNR}_{\text {in }}\left(\mathrm{OSNR}_{\text {in }}<20 \mathrm{~dB}\right)$, when the input signal OSNR provides the dominant noise contribution. At high $\mathrm{OSNR}_{\text {in }}\left(\mathrm{OSNR}_{\text {in }}>30 \mathrm{~dB}\right)$, the idler performance becomes limited by the penalties associated with the conversion, i.e. pump noise transfer due to finite $\nu_{p}$ and CNR, inherent nonlinear distortions, and ASE noise from EDFA post-amplification. During the experiment, the SNR penalty was dependent on the pump power, and varied from $5.2 \mathrm{~dB}$ at $\mathrm{P}_{p}=16 \mathrm{dBm}$ down to $1.8 \mathrm{~dB}$ at $\mathrm{P}_{p}=22 \mathrm{dBm}$ for maximum $\mathrm{OSNR}_{i n}$. The higher pump power (conversion efficiency) effectively reduces the converter loss, and is therefore shown to be beneficial for processing high $\mathrm{OSNR}_{\text {in }}$ signals with low penalty. Nevertheless, the lower pump power is sufficient for low $\mathrm{OSNR}_{\text {in }}$ operation [25]. Note that the measured performance (back-to-back included) saturates due to electrical noise in the transmitter and receiver, and thus diverges from the theoretical maximum at high $\mathrm{OSNR}_{i n}$.

The conversion process and the corresponding limitations are further investigated by analyzing the interaction at the optimum signal launch power that maximizes the received idler SNR. Although OSNR in general does not provide full information on the signal quality, OSNR at the optimum launch power gives more insight into the linear penalties of the converter by including the ASE noise. The simulated OSNR evolution throughout the system around the optimum $\mathrm{P}_{i n}$ is given in Fig. 8 . Both OSNR $_{\text {out }}$ and OSNR $_{r x}$ approach OSNR $_{i n}$, when the input noise is dominant, as also observed in [17]. At high $\mathrm{OSNR}_{\text {in }}, \mathrm{OSNR}_{\text {out }}$ saturates due to pump ASE noise leakage (Fig. 8(a)), and $\mathrm{OSNR}_{r x}$ is further reduced by ASE noise from the EDFA at the converter output (Fig. 8(b)). Increasing pump power leads to increased CE (Fig. 4(b)(c)), and a stronger idler power at the waveguide output, which minimizes OSNR degradation due to pump ASE leakage, and requires less amplification at the converter output. Similar trends as in Fig. 8 have been observed for the corresponding experimental measurements of $\mathrm{OSNR}_{\text {out }}$ and $\mathrm{OSNR}_{r x}$. Throughout the experiment, $\mathrm{OSNR}_{\text {in }}$ includes a single-polarization signal and unpolarized ASE from the EDFA at the converter input. Because of polarization dependent loss of the waveguide, and as FWM occurs predominantly between copolarized signal and pump fields, approximately half of the unpolarized ASE that determines $\mathrm{OSNR}_{\text {in }}$ is effectively suppressed upon conversion. Moreover, the EDFA at the output of the wavelength converter again adds unpolarized ASE to the idler field. This leads to inconsistency in the noise polarization of the experimentally measured $\mathrm{OSNR}_{\text {in }}, \mathrm{OSNR}_{\text {out }}$ and $\mathrm{OSNR}_{r x}$, and therefore numerical simulations with co-polarized signal and noise only have been employed to capture the OSNR evolution instead. It should be noted that for polarization-insensitive converters [11]-[16], the noise would be added uniformly to both signal/idler polarizations.

Finally, the discussed optimum input signal powers are presented in Fig. 9. Given a fixed $\mathrm{OSNR}_{i n}$, the input power interval within $0.2 \mathrm{~dB}$ tolerance of the maximum SNR is found, and the middle of the interval defines the optimum input power values. It is stressed that $\mathrm{P}_{\text {in }}$ is a total signal power that includes signal and noise contributions, depending on a given $\mathrm{OSNR}_{i n}$, and they can be estimated separately from the recorded spectra. The measured optimum total input signal power $\left(\mathrm{P}_{i n}\right)$ and the optimum signal power $\left(\mathrm{P}_{s}\right)$ (excluding noise) is given in Fig. 9(a) and Fig. 9(b), respectively. $\mathrm{P}_{i n}$ and $\mathrm{P}_{s}$ are close to identical at high $\mathrm{OSNR}_{i n}$, but deviate by up to $4 \mathrm{~dB}$ at $\mathrm{OSNR}_{i n}=12 \mathrm{~dB}$ because of the increased noise contribution. The results for the optimum power of the numerical characterization are attached alongside in Fig. 9(c) for reference, and show matching trends. The optimum total power $\mathrm{P}_{i n}$ is approximately independent of $\mathrm{OSNR}_{i n}$, while the optimum signal power $\mathrm{P}_{s}$ drops at low $\mathrm{OSNR}_{i n}$, as observed for both the experimental and 

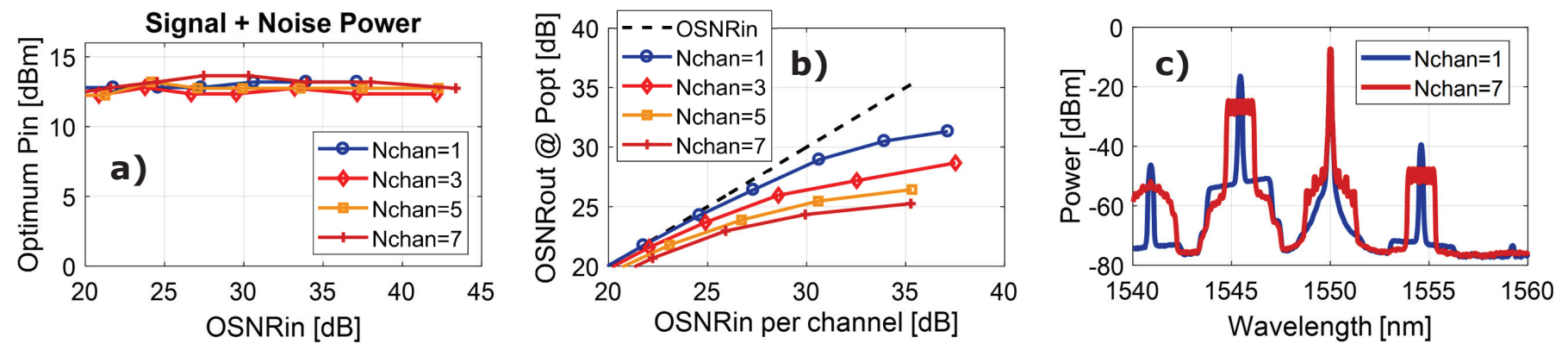

Fig. 10. Experimental characterization of the converter based on a $3 \mathrm{~mm}$ waveguide with a fixed $\mathrm{P}_{p}=20 \mathrm{dBm}$ and a varying number of channels: optimum total input power as a function of total input signal OSNR in (a), per channel idler OSNR at the waveguide output as a function of per channel input signal OSNR (b), and output spectra at optimum launch power for 1 and 7 channels (c).
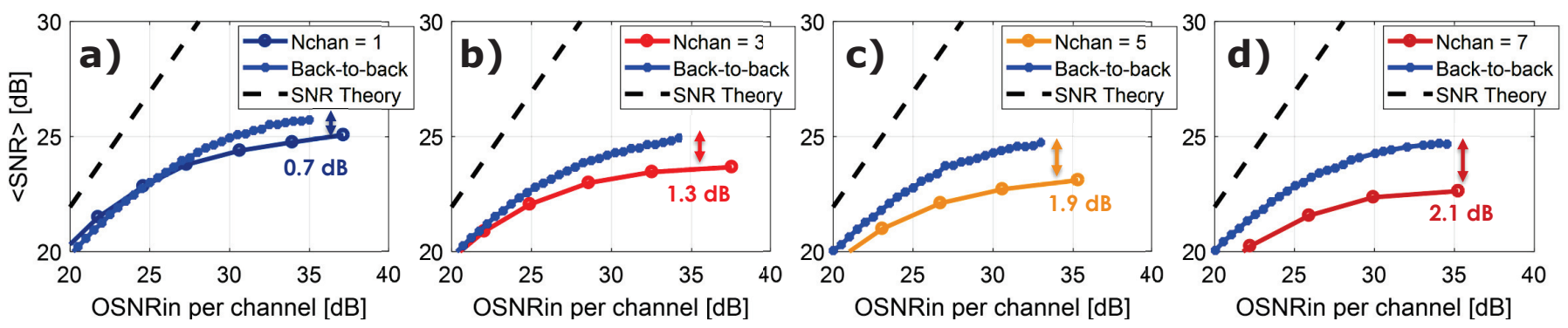

Fig. 11. Maximum received idler SNR at optimum launch power for a $3 \mathrm{~mm}$ waveguide, fixed $\mathrm{P}_{p}=20$ and a varying number of channels.

numerical data. Moreover, it is apparent that the optimum signal launch power decreases with increased $\mathrm{P}_{p}$ to reach a new balance between the linear and the nonlinear penalty, as the idler power is increased due to higher conversion efficiency. Although, the optimum $\mathrm{P}_{\text {in }}$ drops by around $3 \mathrm{~dB}$ as $\mathrm{P}_{p}$ raises from $16 \mathrm{dBm}$ to $22 \mathrm{dBm}$, the net idler power at the waveguide output still increases by almost $10 \mathrm{~dB}$, substantially reducing the required EDFA amplification at the converter output.

\section{Increasing Number of WDM Channels}

The study have so far assumed a constant number of WDM channels under processing, yet the system performance is expected to depend on it as the conversion takes place in a strongly nonlinear medium, where nonlinear interaction between the channels can occur. Throughout the analysis the input power is uniform across all WDM channels, and the measurements are conducted for the central channel only. In the experimental investigation, the input signal quality is varied for a changing number of channels, and the input signal power is optimized for the system with a fixed $\mathrm{P}_{p}=20 \mathrm{dBm}$. Characteristics at the optimum signal launch power for 1, 3, 5 and $7 \mathrm{WDM}$ channels are presented in Fig. 10. Note that we refer to per channel OSNR in Fig. 10(b) to provide a fair comparison between the different WDM systems. The optimum total input power is approximately constant at $\mathrm{P}_{i n}=13 \mathrm{dBm}$ (Fig. 10(a)), meaning that the optimum power per channel decreases with the number of channels due to additional nonlinear penalty from cross-phase modulation (XPM) and inter-channel FWM. The lower optimum power per channel leads to reduced per channel idler OSNR $_{\text {out }}$
(Fig. 10(b)) because of the increased impact of pump noise floor (Fig. 10(c)), which consequently translates into reduction in $\mathrm{OSNR}_{r x}$. Because of the inconsistency in the measured noise polarization discussed in Section IV, OSNR $_{\text {out }}$ at the waveguide output (with ASE noise in one polarization) in Fig. 10(b) was reduced by $3 \mathrm{~dB}$ to compare it to $\mathrm{OSNR}_{i n}$ at the waveguide input (with ASE noise in both polarization).

Ultimately, increased number of channels limits the optimum $\mathrm{OSNR}_{r x}$, and reduces the achievable SNR upon detection. As a result, the conversion penalty increases with the channel count from $0.7 \mathrm{~dB}$ for a single channel up to $2.1 \mathrm{~dB}$ for seven channels, as depicted in Fig. 11. Similar performance decline have been previously reported in [33][35] for FOPA. Moreover, the penalty measured during this experiment does not scale linearly with the number of channels and starts saturating, as also found in [34]. The inter-channel crosstalk can generally be reduced by using a shorter medium with a lower nonlinear coefficient, and a proportionately higher pump power instead [34][37]. The back-to-back measurements have been recorded separately for each case, as the system implementation penalty slightly depends on the number of channels being tested.

\section{Pump Power Optimization}

The experimental pump power sweep from Fig. 7 suggests that increasing $\mathrm{P}_{p}$ will improve the achievable idler SNR due to increased CE. However, the range of powers under test was limited to a maximum of approximately $22 \mathrm{dBm}$ by the burning threshold of the waveguide, resulting in moderate output $\mathrm{CE}$ of around $-15 \mathrm{~dB}$ for 


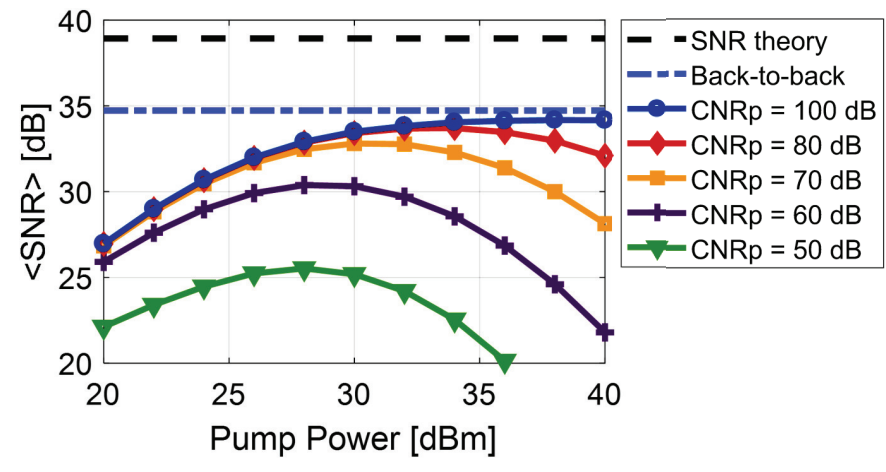

Fig. 12. Simulated maximum received idler SNR as a function of pump power and different input pump carrier-to-noise ratio (CNR) at $\mathrm{OSNR}_{i n}=40 \mathrm{~dB}$ and optimum launch power for a single channel in a $9 \mathrm{~mm}$ waveguide.
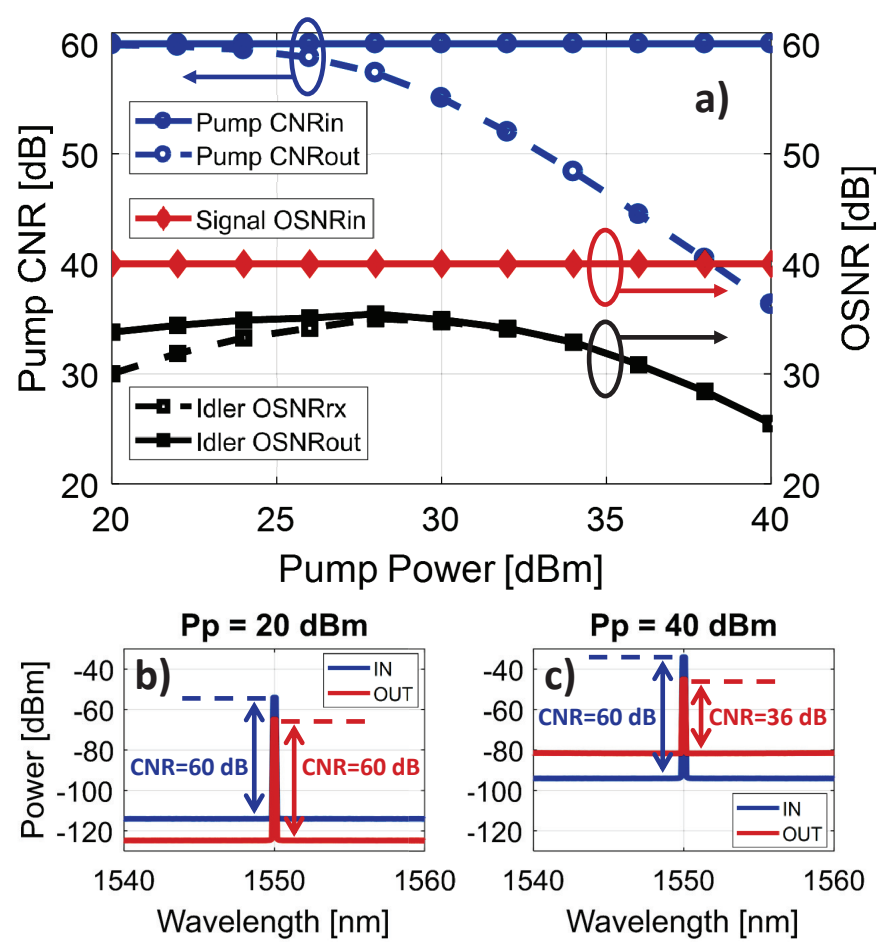

Fig. 13. Simulated pump CNR at the input and output of $9 \mathrm{~mm}$ waveguide with the initial $\mathrm{CNR}=60 \mathrm{~dB}$, and the corresponding evolution of the idler OSNR at optimum input power at the waveguide output and receiver for a single channel at $\mathrm{OSNR}_{i n}=40 \mathrm{~dB}$ (a). Input (blue) and output (red) pump spectra at minimum (b) and maximum (c) pump powers.

$9 \mathrm{~mm}$ waveguide (see Fig. 4(c)). The characterization is extended to higher $\mathrm{P}_{p}$ and $\mathrm{CE}$ by means of numerical simulations, and it also evaluates the impact of the pump noise floor on the system. The noise floor is quantified by means of pump CNR defined as the ratio between the pump power and the noise power in $0.1 \mathrm{~nm}$ bandwidth, as shown in Fig. 3(b). This noise power is uniform in frequency, and identical within and outside the band of the pump. The in-band pump noise leads to amplitude and phase noise transfer from the pump to the idler, and the out-of band noise at idler frequency degrades $\mathrm{OSNR}_{\text {out }}$ of the idler generated on top of it, as discussed in Section IV.
It is noted that the out-of-band pump noise can partially be suppressed by means of optical filtering, as we have also done in the experimental setup from Fig. 3(a). However, due to the trade-off between the filtering extinction ratio and the filtering loss, it is challenging to achieve extremely high CNR levels. Consequently, the residual out-of-band pump noise floor will inevitably impact and degrade the idler performance. The previous study of FOPA in [16], [38] mainly discussed the impact of pump OSNR, assuming the in-band contribution only, and showed increased conversion penalty for the lower pump quality.

This analysis includes both in- and out-of-band pump noise contributions, and is conducted for a single channel at high input signal $\mathrm{OSNR}_{i n}$, as the system has been shown to approach the back-to-back performance for a lower input signal quality. The maximum idler SNR at optimum signal launch power is shown in Fig. 12 as a function of pump power for different values of pump CNR. Increasing $\mathrm{P}_{p}$ to moderate values improves the achievable SNR, but the performance will saturate and deteriorate at high $\mathrm{P}_{p}$. Optimum $\mathrm{P}_{p}$ increases with the initial pump CNR, and the resultant SNR approaches the back-to-back at high CNR. The SNR improvement at low pump powers originates from the increased $\mathrm{CE}$, which effectively leads to higher idler power and reduces the degradations due to pump noise floor and post-conversion EDFA. However, as pump power is further increased, the parametric noise amplification becomes no longer negligible, the pump noise floor raises as discussed in [20], and the output pump CNR is effectively reduced. Consequently, more noise is transferred from the pump to the idler due to increased in-band pump noise, and the generated idler OSNR $_{\text {out }}$ reduces due to the extra out-of-band pump noise leakage. Because the higher $\mathrm{CE}$ will also increase the idler power and require less post-amplification from the EDFA, the idler OSNR $r x$ is further reduced at low pump powers, but will approach OSNR out $_{\text {at }}$ at high $\mathrm{P}_{p}$. The pump CNR and optimum idler OSNR evolution is depicted in Fig. 13 for the initial pump $\mathrm{CNR}=60 \mathrm{~dB}$ and varying $\mathrm{P}_{p}$, alongside the pump spectra at minimum and maximum pump powers. It is shown that the output pump CNR is maintained at $60 \mathrm{~dB}$ at $\mathrm{P}_{p}=20 \mathrm{dBm}$, but drops down to $36 \mathrm{~dB}$ at $\mathrm{P}_{p}=40 \mathrm{dBm}$, leading to increased pump noise transfer and poorer system performance.

\section{Impairments due to Pump Laser Linewidth}

The experimental characterization employed a sub-kHz linewidth fiber laser as pump for FWM to minimize the phase noise transfer. In this final part of the paper, the preliminary analysis from [6] is extended in terms of the impact of a varying pump laser linewidth $\left(\nu_{p}\right)$, and the SNR conversion penalties associated with it are investigated numerically. The results at $\mathrm{OSNR}_{\text {in }}=40 \mathrm{~dB}$ for varying pump powers and two different pump CNR are presented in Fig. 14. The transmitter laser linewidth and the receiver local oscillator linewidth are both set to $10 \mathrm{kHz}$ to emulate the ECLs employed in the experiment. At pump $\mathrm{CNR}=60 \mathrm{~dB}$, the $\mathrm{SNR}$ penalty due to increased 


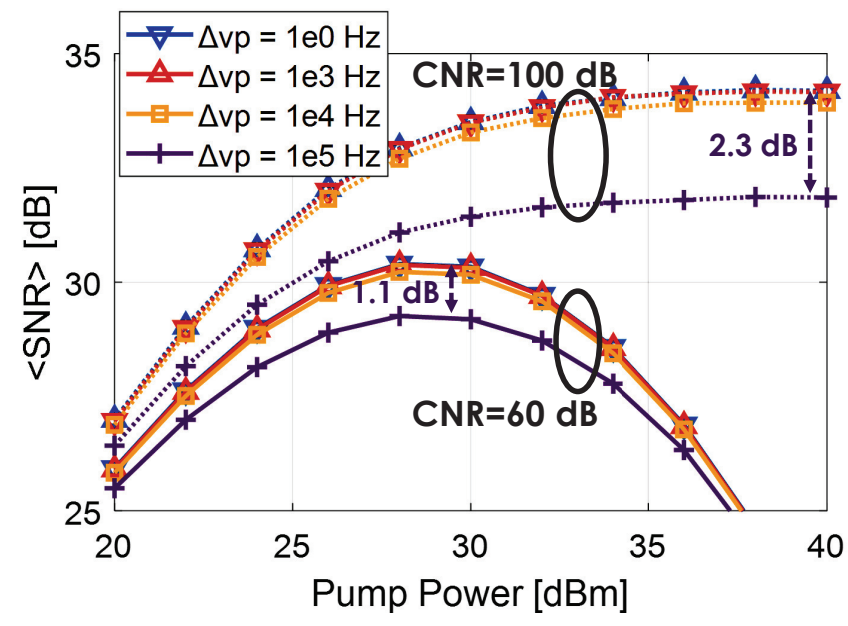

Fig. 14. Simulated maximum received idler SNR as a function of pump power and different pump laser linewidths at pump $\mathrm{CNR}=60 \mathrm{~dB}$ (solid) and $\mathrm{CNR}=100 \mathrm{~dB}$ (dotted) at $\mathrm{OSNR}_{\text {in }}=40 \mathrm{~dB}$ and optimum launch power for a single channel in a $9 \mathrm{~mm}$ waveguide.

$\nu_{p}$ remains below $0.2 \mathrm{~dB}$ for linewidths up to $10 \mathrm{kHz}$, and increases to over $1.1 \mathrm{~dB}$ at $\nu_{p}=100 \mathrm{kHz}$. The penalty due to linewidth will of course change depending on the dominant noise contribution, e.g. it will be more pronounced at high pump CNR (up to $2.3 \mathrm{~dB}$ penalty for $\nu_{p}=100 \mathrm{kHz}$ at $\mathrm{CNR}=100 \mathrm{~dB}$ ) and around optimum $\mathrm{P}_{p}$, and will decrease for noisy input signals.

As the received field for a simple back-to-back setup is a product of the signal and the local oscillator fields [39]: $E_{R X} \propto E_{S} \cdot E_{L O}$, the corresponding total beat-linewidth is then a sum of the transmitter and receiver laser linewidths: $\Delta \nu_{\text {eff }}=\nu_{T X}+\nu_{R X}$, as in [40]. For applications employing a wavelength converter, the wavelength-shifted idler field is a product of the signal and pump fields [22]: $E_{I} \propto E_{S}^{*} \cdot E_{P}^{2}$, thus the effective total beat-linewidth of such systems must also include the pump linewidth contribution: $\Delta \nu_{e f f}=\nu_{T X}+\nu_{R X}+2 \nu_{p}$. This is consistent with the simulated results, as the impact of $\nu_{p}$ remains low for pump linewidths lower than the transmitter and the receiver linewidths $(10 \mathrm{kHz})$, because the total beatlinewidth is dominated by the first two terms originating from the other lasers instead. Consequently, the pump linewidth $\nu_{p} \lesssim\left(\nu_{T X}+\nu_{R X}\right) / 2$ is sufficient for practical applications with negligible penalty.

\section{Conclusion}

In conclusion, this work has characterized the performance of a complete all-optical wavelength conversion system based on degenerated FWM in AlGaAsOI waveguide, and a good correspondence between the experimental and numerical results has been obtained. The approach is general, and can be applied to all converters exploiting the $\chi^{(3)}$ nonlinearity and satisfying the SSFM conditions. SNR instead of OSNR has been employed for performance evaluation in order to capture system impairments beyond ASE noise.
We have shown the importance of the input signal power optimization to reach a balance between the linear and the nonlinear impairments for maximum idler quality, and we have analyzed the performance of the converter at the optimum. The idler quality is limited by the pump noise leakage and ASE from the output EDFA at low input signal powers, and the nonlinear distortions in the waveguide at high input signal powers. We have investigated the impact of the input signal $\mathrm{OSNR}_{\text {in }}$ on the system, and demonstrated negligible conversion penalty for signals dominated by the ASE noise. Moderate pump power $(\mathrm{CE})$ has been shown sufficient for low $\mathrm{OSNR}_{\text {in }}$ operation, while increased pump power has been required for processing high quality inputs with low penalty. The conversion penalty also scales with the number of WDM channels because of the additional nonlinear interaction between them, which limits the allowed input power into the nonlinear medium. Finally, we have performed supplementary numerical characterization on the impact of pump power and pump linewidth, and it has led to identifying the optimum power and the required linewidth of the pump for a specific system.

Ultimately, the analysis has provided a general overview of the complex interaction and performance limits of a FWM-based wavelength converters, outlining practical design guidelines for efficient conversion schemes.

\section{Acknowledgment}

The work was supported by the DNRF Research CoE, SPOC (ref. DNRF123), the DFF project NANOSPECs (DFF-4005-00558B), and the ERC CoG FRECOM (771878).

\section{References}

[1] R. Essiambre and R.W. Tkach, "Capacity Trends and Limits of Optical Communication Networks", Proc. IEEE, 100(5), 1035 1055 (2012).

[2] A.A.M. Saleh and J.M. Simmons, "All-optical networking evolution benefits challenges and future vision", Proc. IEEE, 100(5), 1105-1117 (2012).

[3] C. Lacava, M.A. Ettabib, and P. Petropoulos, "Nonlinear silicon photonic signal processing devices for future optical networks", Applied Sciences, 7(1), 103-107 (2017).

[4] I. Sackey et al., "1.024 Tb/s wavelength conversion in a silicon waveguide with reverse-biased p-i-n junction", Optics Express, 25(18), 21229-21240 (2017).

[5] F. Da Ros et al., "Optical Phase Conjugation in a Silicon Waveguide with Lateral p-i-n Diode for Nonlinearity Compensation", J. Lightwave Technol. 37(2), 323-329 (2019).

[6] F. Da Ros et al., "Characterization and optimization of a highefficiency AlGaAs-On-Insulator-based wavelength converter for 64- and 256-QAM signals", J. Lightwave Technol. 35(17), 37503757 (2017)

[7] M. Pu et al., "Ultra-Efficient and Broadband Nonlinear AlGaAs-on-Insulator Chip for Low-Power Optical Signal Processing", Laser Photon. Rev. 12(12), 1800111 (2018).

[8] M. Pu, L. Ottaviano, E. Semenova, and K. Yvind, "Efficient frequency comb generation in AlGaAs-on-insulator", Optica, 3(8), 823-826 (2016).

[9] L. Ottaviano, M. Pu, E. Semenova, and K. Yvind, "Lowloss high-confinement waveguides and microring resonators in AlGaAs-on-insulator", Optics Letters, 41(17), 3996-3999 (2016). 
[10] H. Hu et al., "Single-source chip-based frequency comb enabling extreme parallel data transmission", Nat. Photonics 12(8), 469473 (2018).

[11] F. Da Ros et al., "Kerr nonlinearity compensation in a 5x28GBd PDM 16-QAM WDM system using fiber-based optical phase conjugation", Proc. ECOC, paper P.5.3, (2014).

[12] A.D. Ellis et al., " $4 \mathrm{~Tb} / \mathrm{s}$ Transmission Reach Enhancement Using $10400 \mathrm{~Gb} / \mathrm{s}$ Super-Channels and Polarization Insensitive Dual Band Optical Phase Conjugation", J. Lightwave Technol. 34(8), 1717-1723 (2016).

[13] H.N. Tan et al., "On the cascadability of all-optical wavelength converter for high-order QAM formats", J. Lightwave Technol. 34(13), 3194-3205 (2016).

[14] I. Sackey et al., "Design and Performance Evaluation of an OPC Device Using a Dual-Pump Polarization-Independent FOPA", Proc. ECOC, paper Tu.1.4.4, (2014).

[15] M.F.C. Stephens, M. Tan, I.D. Phillips, S. Sygletos, P. Harper, and N.J. Doran, "1 THz-Bandwidth Polarization-Diverse Optical Phase Conjugation of 10x114Gb/s DP-QPSK WDM Signals", Proc. OFC, paper W3F.6 (2014).

[16] T. Richter, R. Elschner, A. Gandhe, K. Petermann, and C. Schubert, "Parametric Amplification and Wavelength Conversion of Single- and Dual-Polarization DQPSK Signals", IEEE Journal of Selected Topics in Quantum Electronics 18(2), 988 995 (2012).

[17] H. Kishikawa, N. Goto, and L.R. Chen, "All-optical wavelength preserved modulation format conversion from PDM-QPSK to PDM-BPSK using FWM and interference", J. Lightwave Technol. 34(23), 5505-5515 (2016).

[18] P.O. Hedekvist and P.A. Andrekson, "Noise Characteristics of Fiber-Based Optical Phase Conjugators", J. Lightwave Technol. 17(1), 74-79 (1999).

[19] R. Elschner, "Parametric amplification and wavelength conversion of phase-modulated signals", Doctoral thesis (2011).

[20] P. Kylemark, P.O. Hedekvist, H. Sunnerud, M. Karlsson, and P.A. Andrekson, "Noise characteristics of fiber optical parametric amplifiers", J. Lightwave Technol. 22(2), 409 - 416 (2004).

[21] P. Kylemark, M. Karlsson, and P.A. Andrekson, "Gain and wavelength dependence of the noise-figure in fiber optical parametric amplification", IEEE Photonics Technology Letters, 18(11), 1255 - 1257 (2006).

[22] J. Hansryd, P.A. Andrekson, M. Westlund, J. Li, and P.O. Hedekvist, "Fiber-based optical parametric amplifiers and their applications", IEEE Journal of Selected Topics in Quantum Electronics, 8(3), 506-520 (2002).

[23] P. Kylemark, M. Karlsson, T. Torounidis, and P.A. Andrekson, "Noise statistics of fiber optical parametric amplifiers", J. Lightwave Technol. 25(2), 612 - 620 (2007).

[24] Z. Tong, A. Bogris, M. Karlsson, and P.A. Andrekson, "Full characterization of the signal and idler noise figure spectra in single-pumped fiber optical parametric amplifiers", Optics Express, 18(3), 2884-2893 (2010).

[25] P.M. Kaminski et al., "Signal-to-Idler Conversion Penalty in AlGaAs-on-Insulator Wavelength Converter", Proc. CLEO, paper Stu4C.6. (2018).

[26] G.P. Agrawal, "Nonlinear Fiber Optics", $5^{\text {th }}$ Edition, Elsevier Academic Press, Oxford (2013).

[27] O.V. Sinkin, R. Holzlöhner, J. Zweck, and C.R. Menyuk, "Optimization of the Split-Step Fourier Method in Modeling Optical-Fiber Communications Systems", J. Lightwave Technol. 21(1), 61 - 68 (2003).

[28] T. Richter, R. Nouroozi, H. Suche, W. Sohler, and C. Schubert, "PPLN-Waveguide Based Tunable Wavelength Conversion of QAM Data Within the C-Band", IEEE Photonics Technology Letters, 25(21), 2085 - 2088 (2013).

[29] C. Wang et al., "Ultrahigh-efficiency wavelength conversion in nanophotonic periodically poled lithium niobate waveguides", Optica, 5(11), 1438-1441 (2018).

[30] T. Umeki et al., "Simultaneous nonlinearity mitigation in 92 180Gbit/s PDM-16QAM transmission over $3840 \mathrm{~km}$ using PPLN-based guard-band-less optical phase conjugation", Optics Express, 24(15), 16946-16948 (2016).

[31] M.P. Yankov et al., "Constellation shaping for WDM systems using 256QAM/1024QAM with probabilistic optimization", J. Lightwave Technol. 34(22), 5146-5156 (2016).

[32] R. Essiambre, R.W. Tkach, and R. Ryf, "Fiber Nonlinearity and Capacity: Single-Mode and Multimode Fibers", in I.P.
Kaminow, T. Li, and A.E. Willner, Optical Fiber Telecommunications VIB: Systems and Networks, Elsevier Inc., chapter 1, 1-37, (2013)

[33] I. Sackey et al., "Characterization of a fiber-optical parametric amplifier in a 5 x 28-GBd 16-QAM DWDM system", Proc. OFC, paper W3E.3 (2014).

[34] J.L. Blows, "Design strategy for controlling four-wave mixinginduced crosstalk between channels in a fibre optical parametric amplifier", Opt. Commun. 236(1-3), 115-122 (2004).

[35] M. Jazayerifar et al., "Performance Evaluation of DWDM Communication Systems With Fiber Optical Parametric Amplifiers", J. Lightwave Technol. 31(9), 1454 - 1461 (2013).

[36] M. Jamshidifar, A. Vedadi, and M.E. Marhic, "Reduction of four-wave-mixing crosstalk in a short fiber-optical parametric amplifier", IEEE Photonics Technology Letters 21(17), 12441246 (2009).

[37] R. Elschner, T. Richter, and C. Schubert, "Characterization of FWM-Induced Crosstalk for WDM Operation of a FiberOptical Parametric Amplifier", Proc. ECOC, paper Mo.1.A.2 (2011).

[38] R. Elschner, T. Richter, M. Nölle, J. Hilt, and C. Schubert, "Parametric Amplification of 28-GBd NRZ-16QAM Signals", Proc. OFC, paper OThC2 (2011).

[39] G.P. Agrawal, "Optical Receivers", in Fiber-Optic Communication Systems, 4th Edition, Wiley, chapter 4, 128-181 (2010).

[40] M. Seimetz, "Back-to-Back and Single-Span Transmission", in High-Order Modulation for Optical Fiber Transmission, Springer, chapter 7, 155-204 (2009). 